\title{
The karyotype and sex chromosomes of Praomys tullbergi (Muridae, Rodentia): A detailed characterization
}

\author{
Susana Meles, Filomena Adega, Henrique Guedes-Pinto, Raquel Chaves* \\ Institute for Biotechnology and Bioengineering, Centre of Genetics and Biotechnology, University of Trás-os-Montes \\ and Alto Douro (CGB-UTAD/IBB), 5001-801 Vila Real, Portugal
}

\begin{abstract}
Here we present the first detailed characterization of Praomys tullbergi karyotype, enlightening several chromosome features such as constitutive heterochromatin, telomeric and LINE-1 sequences. The combination of these approaches provided some interesting insights about the genome organization of this African species, which is one of the tullbergi complex elements, a group of species belonging to Murinae (Rodentia, Muridae). Evolutionary considerations on Praomys chromosomes were also achieved, namely, the autosomal complement and the $\mathrm{X}$ chromosome from $P$. tullbergi seem to be derivative chromosomes, most probably resulting from extensive reshufflings during the course of evolution. This conclusion came from the fact that the majority of the chromosomes telomeric sequences are located interstitially, seeming footprints of evolutionary chromosome rearrangements. The detailed analysis of Praomys tullbergi X chromosome suggests that chromosome rearrangements and/or centromere transpositions and addition/elimination of heterochromatin must have been the main evolutionary events that shaped this chromosome.
\end{abstract}

Keywords: Chromosome evolution; Constitutive heterochromatin; LINE-1 sequences; Telomeric sequences; Interstitial telomeric sites

\section{Introduction}

The so-called Praomys group (Rodentia, Muridae) is one of the most diverse and abundant groups of Old World rodents. Praomys tullbergi (PTU) belongs to the tullbergi complex, one of the two species complexes in which the group is divided (Lecompte et al., 2005). The recent literature is not concordant about the number of species comprising the genus; 15 species according to Musser and Carleton (2005), or 11 species according to Lecompte et al. (2005). The lack of information about Praomys raises the importance of a detailed characterization of the group. Dobigny et al. (2002) denote that in the case of African murids, cytogenetic variations provide some of the most reliable diagnostic criteria. Unfortunately, cytogenetic information is lacking for a great part of the recognised Praomys species. No banding or molecular cytogenetic techniques have been applied to chromosomes of P. tullbergi; the only information regarding its chromosomes is a diploid

\footnotetext{
* Corresponding author. Tel.: +351 259350 841; fax: +351 259350572

E-mail address: rchaves@utad.pt (R. Chaves).
}

number of 34, being the sex chromosomes an acrocentric $\mathrm{Y}$ and an acrocentric or metacentric $\mathrm{X}$ chromosomes, according to Matthey (1958) and Granjon et al. (1992) or Capanna et al. (1996), respectively.

Among the different genomic compartments, constitutive heterochromatin $(\mathrm{CH})$, including satellite DNAs and other repetitive sequences have proven to be reliable markers in evolutionary studies (e.g. Saffery et al., 1999; Chaves et al., 2005; Adega et al., 2006). A substantial proportion of the higher eukaryote genome consists of $\mathrm{CH}$, usually residing at the pericentromeric chromosome regions (Chaves et al., 2000). Constitutive heterochromatin and its size differences can be easily identified by the extremely useful technique of Cbanding, but other analytical techniques are indispensable when detailed information on the nature and origin of $\mathrm{CH}$ is required. Among these technologies, base-specific fluorochromes staining (which delimit chromosome regions rich in AT or GC DNA bases) have proven reliability in the understanding of the mechanisms involved in $\mathrm{CH}$ evolution in different genomes (Pieczarka et al., 1998).

Telomeres, the end caps of chromosomes, are specialized functional complexes made up of protein and DNA that not 
only protect the ends of eukaryotic chromosomes (Liu and Fredga, 1999; Blackburn, 2001), but also play an important role in replication, nuclear organization, cellular ageing, tumour progression and gene regulation (Blackburn, 2001). Telomeric DNA consists of sequence motifs of 5-8 bp tandemly repeated, forming long double-stranded homogeneous regions (Henderson, 1995). The (TTAGGG) $)_{n}$ sequences are found mainly at the ends of all vertebrate chromosomes investigated so far, but relatively large blocks of this sequence were already found, in many species, at non-telomeric sites, known as interstitial telomeric sites (ITS) (Liu and Fredga, 1999). The most common ITS are located at the chromosome's pericentric regions (Meyne et al., 1990; de la Sena et al., 1995), but their origin and function are still unclear, although it is considered that such atypically situated telomeric sequences may be related to chromosome rearrangements and thus represent mirror steps of karyotype evolution (Baker and Bickham, 1986; Meyne et al., 1990; de la Sena et al., 1995; Slijepcevic, 1998).

Besides the highly abundant satellite DNA already referred, the mammalian genome also contains repeated sequences interspersed in the euchromatin: the short interspersed repetitive elements (SINEs) and the long interspersed repetitive elements (LINEs) that comprise LINE-1 (or L1), the most abundant and active retrotransposon in the mammalian genome (Boyle et al., 1990; Marchal et al., 2006). LINE-1 sequences in mammals have traditionally been quoted as a selfish element persisting in the genomes due to a replicative advantage over the host genome. Lately this hypothesis has been questioned, and a functional meaning has been addressed to LINE-1 sequences. L1 elements could have acquired some beneficial functions for the host genome as a consequence of parallel coevolution (Britten, 1996a, b), or could play an important role in genome organization and expression at the chromosomal level (Bickmore and Sumner, 1989). The most remarkable example to explain these hypotheses is its supposed implication in $\mathrm{X}$ chromosome inactivation (Bailey et al., 2000; Lyon, 2006). L1 is perhaps the most significant dynamic force operating on the mammalian genome, being the L1 retrotransposition responsible for the generation of new L1, SINE and pseudogene insertions, and causing exon shuffling by transduction of nonL1 sequences (Martin et al., 2005). The high-copy and interspersed nature of L1s and SINEs provide the opportunity for homologous recombination on misaligned chromosomes, leading to translocations, gene duplication and loss (reviewed in Moran and Gilbert, 2002). A structural feature of L1 is a nonrandom physical distribution in the mammalian genome. It was shown that these retroelements are preferentially located at the AT-rich isochores (G-positive bands, late-replicating DNA) of the genome (Boyle et al., 1990), nevertheless a striking and still controversial issue is the special accumulation that they seem to present in the sex chromosomes (Bailey et al., 2000; Waters et al., 2004). Nevertheless, analyses on L1 chromosomal distribution are limited, and few mammalian species have been investigated so far (Marchal et al., 2006).

In the present study, we make a detailed description of $P$. tullbergi(Thomas, 1894) karyotype, enlightening several chromosomal features related with constitutive heterochromatin, telomeric and LINE-1 sequences. A preliminary draft on $P$. tullbergikaryotype and $\mathrm{X}$ chromosome evolution is suggested.

\section{Materials and methods}

\subsection{Chromosome preparations}

A fibroblast cell line from a male $P$. tullbergi (PTU) was obtained from the cell and tissue collection housed at the Department of Systematics and Evolution, Muséum National d'Histoire Naturelle (MNHN). Standard cell culture from PTU was performed as previously described (Chaves et al., 2004a) in order to prepare fixed chromosome spreads.

\subsection{GTD-banding}

Air-dried slides were aged at $658 \mathrm{C}$ overnight and were submitted to standard procedures of G-banding with trypsin. The slides were then fixed with formaldehyde as described by Chaves et al. (2002). Briefly, dry slides were placed in a 1 PBS solution ( $2 \mathrm{~min} 5 \mathrm{~min}$ ) before fixation in $3 \%$ formaldehyde (Sigma)/1 PBS (room temperature). After fixation for $10 \mathrm{~min}$, slides were dehydrated for $5 \mathrm{~min}$ each in $70 \%, 90 \%$ and $100 \%$ chilled ethanol and air dried. At this step, slides were stained with DAPI (instead of routine Giemsa) for a better contrast (Chaves et al., 2002). The inversion of the DAPI colour in Adobe Photoshop revealed the chromosomes G-banding (GTD-banding, G-bands by trypsin with DAPI) for its identification.

\subsection{CBP-banding sequential to G-bands or chromomycin} A3 (CMA3)

The C-banding sequential to G-bands or to chromomycin A3 staining (CMA3) was performed by distaining the slides and submitting them to $\mathrm{C}$-banding technique. CBP-banding (Cbands by barium hydroxide using propidium iodide) was done following the standard procedure of Sumner (1972) but with propidium iodide as a counterstain. Briefly, the slides were submitted to routine C-banding: hydrochloric acid $(0.2 \mathrm{M})$ for $20 \mathrm{~min}$, barium hydroxide (5\% solution) for $7 \mathrm{~min}$ and $2 \mathrm{SSC}$ (saline sodium citrate) at $608 \mathrm{C}, 40 \mathrm{~min}$.

\subsection{DAPI and CMA3 staining}

DAPI (40, $6^{0}$-diamidino-2-phenylindole) and CMA3 (chromomycin A3) staining was performed in fresh fixed chromosome spreads, following routine procedures. The fluorochrome staining was done sequentially, also in combination with the $\mathrm{C}$ banding technique, that is: DAPI + CMA3 + CBP.

\subsection{Fluorescent in situ hybridization (FISH)}

The telomeric probe (TTAGGG) $)_{\mathrm{n}}$ was generated and labelled with digoxigenin-11-dUTP (Roche Molecular Biochemicals) by PCR, as described by Ijdo et al. (1991). The synthetic 
oligonucleotides (TA) 10 were end-labelled also with digoxigenin-11-dUTP by terminal transferase (Roche Molecular Biochemicals), following the manufacturer's instructions. Whole chromosome paint probes from Mus musculus (MMU) representative of the $\mathrm{X}$ and $\mathrm{Y}$ chromosomes were purchased at Chrombios $\mathrm{GmbH}$ and were labelled with digoxigenin-11-dUTP (Roche Molecular Biochemicals) or biotin-16-dUTP (Sigma). Finally, the LINE-1 probe was synthesized by PCR from the $P$. tullbergi genome and fluorescently labelled with digoxigenin-11-dUTP (Roche Molecular Biochemicals). The primers used were the ones described by Deuve et al. (2006) and amplified a 290 bp DNA fragment. Labels were detected by FITC conjugated with avidin (Vector Laboratories) and anti-digoxigenin-rhodamine (Roche Molecular Biochemicals). All the FISH procedures were the standard ones.

\subsection{Chromosome observation}

Chromosomes were observed with a Zeiss Axioplan 2 Imaging microscope coupled to an Axiocam digital camera and AxioVision software (Version 2.0.5-Zeiss). Digitised photos were prepared for printing in Adobe Photoshop (Version 5.0); contrast and colour optimisation were the functions used and all affected the whole of the image equally.

\section{Results}

\subsection{G- and C-banding}

Cytogenetic analysis of $P$. tullbergi (PTU) revealed a karyotype composed by 16 acrocentric autosomes (Fig. 1a and b), being the sex chromosomes a large submetacentric $\mathrm{X}$ and an acrocentric Y (Fig. 1a and b). In Fig. 1c and d is possible to observe that most of the autosome constitutive heterochromatin $(\mathrm{CH})$ is centromeric, however some interstitial and telomeric $\mathrm{C}$ bands can be distinguished in a few chromosomes (e.g. chromosomes 16 and 10, respectively). The sex chromosomes are the ones presenting the higher amount of $\mathrm{CH}$, namely, the $\mathrm{X}$ chromosome presents six $\mathrm{CH}$ regions, of which one is a prominent pericentromeric $\mathrm{C}$-band in the $\mathrm{p}$-arm and five are interstitial, one of which is a huge C-band in the q-arm. The $\mathrm{Y}$ chromosome is largely heterochromatic being even difficult to discriminate any differential staining (see Fig. 1d). Single Cbanding was also performed in these chromosomes revealing no significant differences from the observed C-banding after Gbanding.

\subsection{Fluorochrome staining}

The application of DNA-specific fluorescent dyes was performed in order to ascertain the affinity to AT- or GC-rich regions in $P$. tullbergi chromosomes (Fig. 2).

In a broad observation, DAPI staining revealed a Gbanding like pattern in all autosomes as well as in the $\mathrm{X}$ chromosome (cf. Fig. 2d with Fig. 1b).
The Y chromosome did not show any longitudinal differentiation; however it was possible to observe a homogeneous bright fluorescent staining in the whole chromosome, indicative of a preferential AT base composition (Fig. 2d).

CMA3 revealed a bright fluorescence in the majority of the $\mathrm{C}$-positive regions and in some chromosomes it is even possible to observe a less pronounced R-like banding (e.g. Fig. $2 \mathrm{~d}$, chromosome 2). In the $\mathrm{Y}$ chromosome a small GCenriched terminal region was detected (Fig. 2d).

The sequential C-banding (Fig. 2d) enabled to discriminate the molecular composition (AT, GC or AT $+\mathrm{GC}$ ) of $P$. tullbergi $\mathrm{CH}$ regions, which is referred (identified) next to the bands in Fig. 2. In the autosomes, the telomeric $\mathrm{CH}$ demonstrated to be of AT or GC nature, and the interstitial Cbands GC or AT + GC. In the X chromosome it was possible to discriminate the interstitial $\mathrm{C}$-bands into two $\mathrm{CH}$ subclasses: $\mathrm{AT}+\mathrm{GC}$ and GC.

\subsection{Telomeric in situ hybridization sequences}

The chromosomes of $P$. tullbergi showed discrete telomeric hybridization signals on both chromatids at the telomere domains in all the chromosomes (Fig. 3a and b). Moreover, a large number of acrocentric autosomes also displayed interstitial telomeric sites (ITS) located at pericentromeric (e.g. PTU 11 and PTU15 in Fig. 3b) and/or interstitial regions (e.g. PTU6 and PTU9 in Fig. 3b) and this was also observed in the $\mathrm{X}$ chromosome (Fig. 3d). This evidence was confirmed by in situ hybridization with the oligonucleotide (TA $)_{10}$ in PTU chromosomes (Fig. 3c and d), that revealed essentially similar in situ hybridization signals to the ones obtained with the telomeric vertebrate probe.

\subsection{Further analysis of PTU sex chromosomes with MMUX, MMUY and LINE-1 probes}

In order to increase our current knowledge on the sex chromosomes, painting probes from M. musculus $\mathrm{X}$ and $\mathrm{Y}$ chromosomes and the LINE-1 probe prepared from PTU were in situ hybridized to PTU chromosome spreads (Fig. 4). In Fig. $4 \mathrm{a}$ it is possible to observe that the MMUX paint probe produces at least, three syntenic blocks in PTUX chromosome; the regions not hybridized by this probe are the major C-bands already described above in the PTUX chromosome. The MMUY paint probe disclosed only a small syntenic block in the PTUY chromosome (Fig. 4b), what is not surprising since this chromosome revealed to be extremely heterochromatic.

Finally, as referred, we also analysed LINE-1 distribution on PTU chromosomes (Fig. 4c) with the aim to observe an eventual privileged accumulation of this sequence on the $\mathrm{X}$ chromosome, as described by some authors (e.g. Lyon, 2006; Marchal et al., 2006). Analysis of Fig. 4c shows that this sequence is not found in the centromeric heterochromatin blocks neither in the interstitial heterochromatic regions of the autosomes. The sex $\mathrm{X}$ chromosome showed these same hybridization features, and the $\mathrm{Y}$ chromosome demonstrated 
a preferential accumulation of LINE-1 sequences at the terminal region (syntenic region of the MMUY probe, cf. Fig. 4b). Moreover, the LINE-1 probe produces an almost longitudinal differentiation of the chromosomes capable of a banding pattern, with a privileged location at the AT-rich isochores. There was no preferential accumulation of LINE-1 sequences at the sex chromosomes compared to the autosomal complement.

Finally, in Fig. 5 we present a compilation of all the results (Fig. 5a-e) concerning the sex chromosomes and respective ideograms (Fig. 5f) for a straightforward discussion of all data.

\section{Discussion}

\subsection{Karyotype features of P. tullbergi}

The cytogenetic characterization provides some of the most reliable taxonomic criteria (Dobigny et al., 2002; Seuánez et al., 2005) but this is still scarce for most of the recognised Praomys species. In the current work, we present the first detailed documented characterization of P.tullbergi(PTU) karyotype (one of the Praomys genus species), using classical and molecular cytogenetic tools that allowed to draw a compre- hensive and clear cytogenetic picture of this species.

The PTU karyotype is essentially acrocentric with 16 acrocentric autosome pairs, a large submetacentric $\mathrm{X}$ and an acrocentric Y chromosome (Fig. 1). C-banding technique revealed that most of the autosome constitutive heterochromatin $(\mathrm{CH})$ is located at the centromeric regions; however it is possible to disclose interstitial and telomeric $\mathrm{CH}$ regions (Fig. 1). The sex chromosomes are the ones presenting the higher amount of $\mathrm{CH}$, being the $\mathrm{Y}$ chromosome essentially heterochromatic (Fig. 1).

DNA-specific fluorescent dyes (DAPI + CMA3) plus Cbanding allows an expedite characterization of $\mathrm{CH}$ (regarding its location, detection of different subclasses and revelation of its molecular composition) much more accurately than the classical single C-banding (that only reveals the localization of the major $\mathrm{CH}$ blocks). The application of such DNA-specific fluorescent dyes resulted in an enhanced characterization of the DNA base composition of PTU karyotype (Fig. 2), as well as a $\mathrm{CH}$ class categorization. With this methodology it was possible to find, at least, five $\mathrm{CH}$ classes in the autosomal complement: one centromeric, two interstitial (GC and AT + GC nature) and three telomeric $\mathrm{CH}$ classes (GC, AT and AT + GC composition). The $\mathrm{X}$ chromosome revealed, at least, three $\mathrm{CH}$ classes: one pericentromeric and two interstitial (AT + GC and GC nature). The present work demonstrated that the application of DNA-specific fluorescent dyes and sequential C-banding (Fig. 2) could be an alternative tool for the analysis of rodent chromosomes constitutive heterochromatin heterogeneity, especially when other specific techniques, as fluorescent in situ hybridization with repetitive DNA sequences, are not available. Since the presence of $\mathrm{CH}$ facilitates the occurrence of chromosomal rearrangements, as in accordance with several authors (Yunis and Yasmineh, 1971; Chaves et al., 2004b), its analysis should be a helpful tool in evolutionary studies.
Constitutive heterochromatin characterization of $P$. tullbergi chromosomes demonstrated diversity, not only in what concerns location, but also in the existence of subclasses presenting different molecular natures.

In what concerns the analysis of telomeric sequences in $\mathrm{P}$. tullbergi, these were predominantly found at the physical ends of the chromosomes, as expected (Fig. 3), but several discrete interstitial telomeric repeats (ITS) were also detected in various autosomes and in the $\mathrm{X}$ chromosome. Chromosomes of many vertebrate species exhibit ITS, most of them located pericentromerically within regions of constitutive heterochromatin (Meyne et al., 1990). It has been suggested that these ITSs occurred as a result of Robertsonian-like fusions and fissions (Slijepcevic, 1998), or tandem chromosome fusions (Li et al., 2000) during karyotype evolution. For instance, in some rodents, most of the ITSs found are located pericentromerically as a result of Robertsonian translocations (e.g. Fagundes et al., 1997; Faravelli et al., 1998; Andrades-Miranda et al., 2002; Castiglia et al., 2006). Dobigny et al. (2003) reported ITSs located at interstitial chromosome regions in Taterillus sp. resulting from tandem fusions. In contrast, Robertsonian exchanges in the house mouse showed complete loss of the telomeres (Garagna et al., 1995; Nanda et al., 1995). Some of these ITSs co-localize with Cbands, whilst others does not (data showed for the $\mathrm{X}$ chromosome, Fig. 5). Several hypotheses have been addressed in the literature to explain how these interstitial telomeres present in the chromosomal arms may have lost their function and become inactive (see for instances Slijepcevic, 1998). Nevertheless, the conservation or, at least, partial conservation of these telomeric repeats can be explained by their inactivation, thus favouring true end-to-end fusions (Slijepcevic, 1998). An alternative explanation is that only degenerated telomeric repeats are observed, suggesting the presence of subtelomeric arrays rather than true and formerly functional TTAGGG repeats (Slijepcevic, 1998). It is also possible that ITSs may reflect saltatory replication of telomeric sequences from inactive centromeric regions of compound chromosomes (Pathak, 1995). This specific explanation can be illustrated by satellite DNA families exhibiting telomeric repeats integrated in the original sequence, as it is the case of FA-SAT from Felis catus (Fanning, 1987; Santos et al., 2004) and for ITSs that co-localize with C-bands (as a great amount of $\mathrm{CH}$ is composed of centromeric satellite DNA sequences). Therefore, and from the referred above, it seems that karyotypes and chromosomes in a more primitive evolutionary state would contain telomeric repeats solely located at the telomeres (Meyne et al., 1990). In this way it is reasonable to consider PTU karyotype a derivative one, as it is possible to detect ITSs at several chromosome arms. Furthermore, these ITSs most probably are the result of tandem fusions, since the majority of them are located at interstitial chromosomal regions, as described in Taterillus sp. by Dobigny et al. (2003). This hypothesis is currently being verified in our lab by comparative chromosome painting. In what concerns the telomeric repeats located pericentromerically, they do not seem likely to be the result of Robertsonian translocations, given that the PTU karyotype is acrocentric. 
The integration of telomeric repeats by saltatory replication in the centromeric satellite DNA seems a more probable scenario, even more because these ITSs are $\mathrm{C}$ positive. This assumption needs further support, namely with physical mapping of satellites isolated from PTU genome, particularly the ones located at the centromeres. Nevertheless, other explanations can be drawn, as for example, the occurrence of small pericentric inversions.

Although the origin and function of ITS still remains unclear, it is considered, as referred, that such atypically situated telomeric sequences may be related to chromosomal rearrangements and thus representing mirror steps of karyotype evolution (Baker and Bickham, 1986; Meyne et al., 1990; de la Sena et al., 1995; Slijepcevic, 1998). From our results, we believe that the telomeric repeats detected pericentromeric and interstitially in several chromosomes of P.tullbergikaryotype are nonambiguous footprints of evolutionary chromosome rearrangements.

\subsection{The sex chromosomes of P. tullbergi}

To obtain a comprehensive analysis of $P$. tullbergi sex chromosomes, $\mathrm{X}$ and $\mathrm{Y}$ whole chromosome paint probes from M. musculus were in situ hybridized to PTU chromosome spreads. Simultaneously the genome was analysed regarding LINE-1 retrotransposition sequences (Fig. 4). Fig. 5 shows a collection of all the results on Praomys sex chromosomes.

The X chromosome from M. musculus (MMUX) delineated, at least, three syntenic segments in the submetacentric PTUX chromosome. These three syntenic segments seem to comprise three small $\mathrm{CH}$ regions in the PTU q-arm (cf. Fig. 5f), probably due to an artefact of the fluorescent signal expansion, since painting probes are enriched in single copy DNA sequences. MMUY probe revealed in PTUY only a small syntenic region in the terminal q-arm (Fig. 5e and f). To some extent this was an expected result, since the PTUY chromosome is essentially heterochromatic (Figs. 1d and 5e).

The sex chromosomes from Rodentia sp. are extremely diverse, display different contents of heterochromatin, and is even frequent to find species with autosomal/X-chromosome translocations (e.g. Deuve et al., 2006). The comparative analysis of the $\mathrm{X}$ chromosomes from Rattus norvegicus (RNO) and M. musculus in the syntenic view of the Ensembl database (www.ensembl.org) demonstrated that both acrocentric $\mathrm{X}$ chromosomes are extremely rearranged, being separated by, at least, two major inversions, with other small segments involved in other rearrangements. The direct comparison of PTUX (submetacentric, six $\mathrm{CH}$ regions, Fig. 5) with MMUX (acrocentric, two C-bands of which one centromeric and other interstitial, data not shown) and RNOX chromosomes (acrocentric, two C-bands of which one centromeric and other interstitial, data not shown) clearly shows differences in respect to morphology and heterochromatin content, suggesting that chromosome rearrangements and/or centromere transpositions and addition/elimination of heterochromatin must have been involved in the evolution of these chromosomes. The detection of ITSs in PTUX (Fig. 5f) is also indicative of chromosomal rearrangements occurrence, as they may represent remnant DNA sequences from the ancestor telomeres. Moreover, and as referred, the "Synteny view" comparative analysis (genome bowser Ensembl (www.ensembl.org)) between MMUX and RNOX lead us to suggest that PTUX chromosome is most probably the result of a much more complex evolutionary scenario requiring further investigation.

At present, there is strong evidence for an accumulation of LINE-1 on the $\mathrm{X}$ chromosome in such a distribution that could fulfil the function of booster elements in XCI (Lyon, 2006); whether or not L1 have indeed such a function, is less clear (Lyon, 2006). Moreover, L1 is a source of genetic diversity due to changes in gene expression, location and organization, which is considered fundamental for genome plasticity and hence, evolution (Kidwell, 2002). Based on these assumptions, we analysed the distribution and relative chromosome density of LINE-1 sequences in PTU chromosomes (Figs. 4c and 5d and f). LINE-1 sequences were observed with a preferential location at the AT-rich isochores and showed a ubiquitous chromosome distribution resembling a banding pattern, known to correspond to G-positive regions (AT-rich isochores) (cf. Fig. 5d, and see e.g. Boyle et al., 1990). The centromeric and interstitial heterochromatic blocks did not show the presence of this sequence. Furthermore, we did not detect any preferential accumulation of LINE-1 sequences in the sex chromosomes, when compared with the autosomal complement, what is somehow surprising when confronted with other works already referred. Curiously, the $\mathrm{Y}$ chromosome revealed an accumulation of these sequences at the terminal region (exactly in the syntenic region of MMUY). Importantly, and in marked contrast to the distribution shown in most mammals (Boyle et al., 1990; Bailey et al., 2000; Dobigny et al., 2004; Waters et al., 2004; Ross et al., 2005) and particularly in some other rodents (e.g. Casavant et al., 2000; Deuve et al., 2006), there was no enrichment on the $\mathrm{X}$ chromosome of $\mathrm{P}$. tullbergi. The finding of a general chromosome distribution of LINE-1 in some species could be an important issue in the ascertainment of the exact role of these elements in X-chromosome inactivation and should therefore, be further analysed.

\subsection{Concluding remarks}

Although the order Rodentia is the most speciose of all mammals, with more than 2000 recognized species (Wilson and Reeder, 2005), few karyotypic and chromosome studies have been undertaken in the different taxa. At present, a time where the rodent chromosome evolution field is experiencing a great expansion, the karyological knowledge should be the foundation to any genomic research.

As far as we know, this is the first detailed characterization of $P$. tullbergi karyotype. Our data demonstrates that the autosomal complement as well as the $\mathrm{X}$ chromosome from $P$. tullbergi are derivative, and must have been extremely rearranged during the course of evolution. This idea is supported by the $\mathrm{CH}$ characterization and the ITSs detected 
on these chromosomes, which point to the occurrence of evolutionary reshufflings and may represent a suitable tool in reconstructing the path of karyotypic rearrangements and assisting in the inference of ancestral chromosomes and karyotype forms. Finally, the level of rearrangements implicated in P.tullbergichromosome evolution suggested by our results evidences the absolute need for a comparative genomics analysis of the karyotype with more robust molecular tools.

\section{Acknowledgements}

This work was supported by the project POCI/BIA-BCM/ $58541 / 2004$ and a post-doc grant SFRH/BPD/32661/2006 of the Science and Technology Foundation (FCT) from Portugal. We are deeply grateful to Dr. Vitaly Volobouev for providing the cell cultures of $P$. tullbergi and for the revision of this manuscript.

\section{References}

Adega, F., Chaves, R., Guedes-Pinto, H., Heslop-Harrison, J.S., 2006. Physical organization of the 1.709 satellite IV DNA family in Bovini and Tragelaphini tribes of the Bovidae: sequence and chromosomal evolution. Cytogenet. Genome Res. 114, 140-146.

Andrades-Miranda, J., Zanchin, N.I., Oliveira, L.F., Langguth, A.R., Mattevi, M.S., 2002. $\left(\mathrm{T}_{2} \mathrm{AG}_{3}\right)_{\mathrm{n}}$ telomeric sequence hybridization indicating centric fusion rearrangements in the karyotype of the rodent oryzomys subflavus. Genetica 114 (1), 11-16.

Bailey, J.A., Carrel, L., Chakravarti, A., Eichler, E.E., 2000. Molecular evidence for a relationship between LINE-1 elements and X chromosome inactivation: the Lyon repeat hypothesis. Proc. Natl. Acad. Sci. U.S.A. 97, 66346639.

Baker, R.J., Bickham, J.W., 1986. Speciation by monobrachial centric fusions. Proc. Natl. Acad. Sci. U.S.A. 83, 8245-8248.

Bickmore, W.A., Sumner, A.T., 1989. Mammalian chromosome banding an expression of genome organization. Trends Genet. 5, 144-148.

Blackburn, E.H., 2001. Switching and signaling at the telomere. Cell 106, 661673.

Boyle, A.L., Ballard, S.G., Ward, D.C., 1990. Differential distribution of long and short interspersed element sequences in the mouse genome: chromosome karyotyping by fluorescence in situ hybridization. Proc. Natl. Acad. Sci. U.S.A. 87, 7757-7761.

Britten, R.J., 1996a. Cases of ancient mobile element DNA insertions that now affect gene regulation. Mol. Phylogenet. Evolut. 5, 13-17.

Britten, R.J., 1996b. DNA sequence insertion and evolutionary variation in gene regulation. Proc. Natl. Acad. Sci. U.S.A. 93, 9374-9377.

Capanna, E., Codjia, J.T.C., Chrysostome, C., Civitelli, M.V., 1996. Les chromosomes des Rongeurs du Bénin (Afrique de l'Ouest): 3 Murinae. Atti della Accademia dei lincei classe di scienze fisiche matematiche e naturali rendiconti lincei scienze fisiche e naturali, serie 8 (9), 25-37.

Casavant, N.C., Scott, L., Cantrell, M.A., Wiggins, L.E., Baker, R.J., Wichman, H.A., 2000. The end of LINE? Lack of recent L1 activity in a group of South American Rodents. Genetics 154, 1809-1817.

Castiglia, R., Garagna, S., Merico, V., Oguge, N., Corti, M., 2006. Cytogenetics of a new cytotype of African Mus (subgenus Nannomys) minutoides (Rodentia, Muridae) from Kenya: C- and G-banding and distribution of (TTAGGG)n telomeric sequences. Chromosome Res. 14, 587-594.

Chaves, R., Guedes-Pinto, H., Heslop-Harrison, J.S., Schwarzacher, T., 2000. The species and chromosomal distribution of the centromeric a-satellite I sequence from sheep in the tribe Caprini and other Bovidae. Cytogenet. Cell Genet. 91, 62-66.
Chaves, R., Adega, F., Santos, S., Heslop-Harrison, J.S., Guedes-Pinto, H., 2002. In situ hybridization and chromosome banding in mammalian species. Cytogenet. Genome Res. 96, 113-116.

Chaves, R., Frönicke, L., Guedes-Pinto, H., Wienberg, J., 2004a. Multidirectional chromosome painting between the Hirola antelope (Damaliscus hunteri, Alcelaphini, Bovidae), sheep and human. Chromosome Res. 12, 495-503.

Chaves, R., Santos, S., Guedes-Pinto, H., 2004b. Comparative analysis (Hippotragini versus Caprini, Bovidae) of X-chromosome's constitutive heterochromatin by in situ restriction endonuclease digestion: X-chromosome constitutive heterochromatin evolution. Genetica 121, 315-325.

Chaves, R., Guedes-Pinto, H., Heslop-Harrison, J.S., 2005. Phylogenetic relationships and the primitive $\mathrm{X}$ chromosome inferred from chromosomal and satellite DNA analysis in Bovidae. Proc. Roy. Soc. B 272, 2009-2016.

de la Sena, C., Chowdhary, B.P., Gustavsson, I., 1995. Localization of the telomeric (TTAGGG) $)_{n}$ sequences in chromosomes of some domestic animals by fluorescence in situ hybridization. Hereditas 123, 269-274.

Deuve, J.L., Bennett, N.C., O’Brien, P.C.M., Fergunson-Smith, M.A., Faulkes, C.G., Britton-Davidian, J., Robinson, T.J., 2006. Complex evolution of X and $\mathrm{Y}$ autosomal translocations in the giant mole-rat. Cryptomys mechowi (Bathyergidae). Chromosome Res. 14, 681-691.

Dobigny, G., Baylac, M., Denys, C., 2002. Geometric morphometrics, neural networks and diagnosis of sibling Taterillus (Rodentia, Gerbillinae). Biol. J. Linn. Soc. 77 (3), 319-327.

Dobigny, G., Ozouf-Costaz, C., Bonillo, C., Volobouev, V., 2003. Evolution of rRNA gene clusters and telomeric repeats during explosive genome repatterning in Taterillus X (Rodentia, Gerbillinae). Cytogenet. Genome Res. 103, 94-103.

Dobigny, G., Ozouf-Costaz, C., Waters, P.D., Bonillo, C., Coutanceau, J.P., Volobouev, V., 2004. LINE-1 amplification accompanies explosive genome repatterning in rodents. Chromosome Res. 12, 787-793.

Fagundes, V., Vianna-Morgante, A.M., Yonenaga-Yassuda, Y., 1997. Telomeric sequences localization and G-banding patterns in the identification of a polymorphic chromosomal rearrangement in the rodent Akodon Cursor $(2 \mathrm{n}=14,15$ and 16). Chromosome Res. 5, 228-232.

Fanning, T., 1987. Origin and evolution of a major feline satellite DNA. J. Mol. Biol. 197, 627-634.

Faravelli, M., Moralli, D., Bertoni, L., Attolini, C., Chernova, O., Raimondi, E., Giulotto, E., 1998. Two extended arrays of a satellite DNA sequence at the centromere and at the short-arm telomere of Chinese hamster chromosome 5. Cytogenet. Cell Genet. 83, 281-286.

Garagna, S., Broccoli, D., Redi, C.A., Searle, J.B., Cooke, H.J., Capanna, E., 1995. Robertsonian metacentrics of the house mouse lose telomeric sequences but retain some minor satellite DNA in the pericentromeric area. Chromosoma 103 (10), 685-692.

Granjon, L., Duplantier, J.M., Catalan, J., Britton-David, J., 1992. Karyotypic data on rodents from Senegal. J. Zool. 38 (3-4), 263-276.

Henderson, E., 1995. Telomere DNA structure. In: Blackburn, E.H., Greider, C.E. (Eds.), Telomeres. Cold Spring Harbour Laboratory Press, Cold Spring, pp. 11-34.

Ijdo, J.W., Wells, R.A., Baldini, A., Reeders, S.T., 1991. Improved telomere detection using a telomere repeat probe (TTAGGG) $)_{n}$ generated by PCR. Nucleic Acids Res. 19 (17), 478.

Kidwell, M.G., 2002. Transposable elements and the evolution of genome size in eukaryotes. Genetica 115, 49-63.

Lecompte, E., Denys, C., Granjon, L., 2005. Confrontation of morphological and molecular data: The Praomys group (Rodentia, Murinae) as a case of adaptive convergences and morphological stasis. Mol. Phylogenet. Evolut. 37, 899-919.

Li, Y.-C., Lee, C., Sanoudou, D., Hsu, T.-H., Li, S.-Y., Lin, C.-C., 2000. Interstitial colocalization of two cervid satellite DNAs involved in the genesis of the Indian muntjac karyotype. Chromosome Res. 8, 363-373.

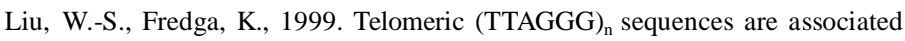
with nucleolus organizer regions (NORs) in the wood lemming. Chromosome Res. 7, 235-240. 
Lyon, M.F., 2006. Do LINEs have a role in X-chromosome inactivation? J. Biomed. Biotechnol. 1-6.

Marchal, J.A., Acosta, M.J., Bullejos, M., Puerma, E., Díaz de la Guardia, R., Sánchez, A., 2006. Distribution of L1-retroposons on the giant sex chromosomes of Microtus cabrerae (Arvicolidae, Rodentia): functional and evolutionary implications. Chromosome Res. 14, 177-186.

Martin, S.L., Li, W-L.P., Furano, A.V., Boissinot, S., 2005. The structures of mouse and human L1 elements reflect their insertion mechanism. Cyto- genet. Genome Res. 110, 223-228.

Matthey, R., 1958. Les chromosomes et la position systématique de quelques Murinae africains (Mammalia-Rodentia). Acta Tropica 15, 97-117.

Meyne, J., Baker, R.J., Hobart, H., Hsu, T.C., Ryder, O.A., Ward, O.G., Wiley, J.E., Wurster-Hill, D.H., Yates, T.L., Moyzis, R.K., 1990. Distribution of non-telomeric sites of the (TTAGGG) $)_{n}$ telomeric sequence in vertebrate chromosomes. Chromosoma 99, 3-10.

Moran, J.V., Gilbert, M., 2002. Mammalian LINE-1 retrotransposons and related elements. In: Craig, N.L., Craigie, R., Gellert, M., Lambowitz, A.M. (Eds.), Mobile DNA II. ASM Press, Washington, DC, pp. 836-869.

Musser, G., Carleton, M., 2005. Superfamily Muroidea. In: Wilson, D.E., Reeder, D.M. (Eds.), Mammal Species of the World. Smithsonian Institution Press, Washington, DC.

Nanda, I., Schneider-Rasp, S., Winking, H., Schmid, M., 1995. Loss of telomeric sites in the chromosomes of Mus musculus domesticus (Rodentia, Muridae) during Robertsonian rearrangements. Chromosome Res. 3, 399-409.

Pathak, S., 1995. Centromere or telomere: who is the boss? Anticancer Res. 15, 2549-2550.
Pieczarka, J.C., Nagamachi, C.Y., Muniz, J.A.P.C., Barros, R.M.S., Mattevi, M.S., 1998. Analysis of constitutive heterochromatin of Aotus (Cebidae, Primates) by restriction enzyme and fluorochrome bands. Chromosome Res. 6, 77-83.

Ross, M.T., Grafham, D.V., Coffey, A.J., et al., 2005. The DNA sequence of the human X chromosome. Nature 434 (7031), 325-337.

Saffery, R., Earle, E., Irvine, D.V., Kalitsis, P., Choo, K.H., 1999. Conservation of centromere protein in vertebrates. Chromosome Res. 7 (4), 261-265.

Santos, S., Chaves, R., Guedes-Pinto, H., 2004. Chromosomal localization of the major satellite DNA family (FA-SAT) in the domestic cat. Cytogenet. Genome Res. 107, 119-122.

Seuánez, H.N., Bonvicino, C.R., Moreira, M.A., 2005. The primates of the Neotropics: genomes and chromosomes. Cytogenet. Genome Res. 108 (13), 38-46.

Slijepcevic, P., 1998. Telomeres and mechanisms of Robertsonian fusion. Chromosoma 107, 136-140.

Sumner, A.T., 1972. A simple technique for demonstrating centromeric heterochromatin. Exp. Cell Res. 75, 304-306.

Thomas, O., 1894. Note on Mus burtoni, Thos. Ann Mag. Nat. History 8 (6), 204.

Waters, P.D., Dobigny, G., Pardini, A.T., Robinson, T.J., 2004. LINE-1

dis- tribution in Afrotheria and Xenarthra: implications for understanding the evolution of LINE-1 in eutherian genomes. Chromosoma 113 (3), 137-144.

Wilson, D.E., Reeder, D.M., 2005. In: Mammal Species of the World: A Taxonomic and Geographic Reference, 3rd ed. The Johns Hopkins University Press, Baltimore.

Yunis, J.J., Yasmineh, W.G., 1971. Heterochromatin, satellite DNA and cell function. Science 174, 1200-1209. 


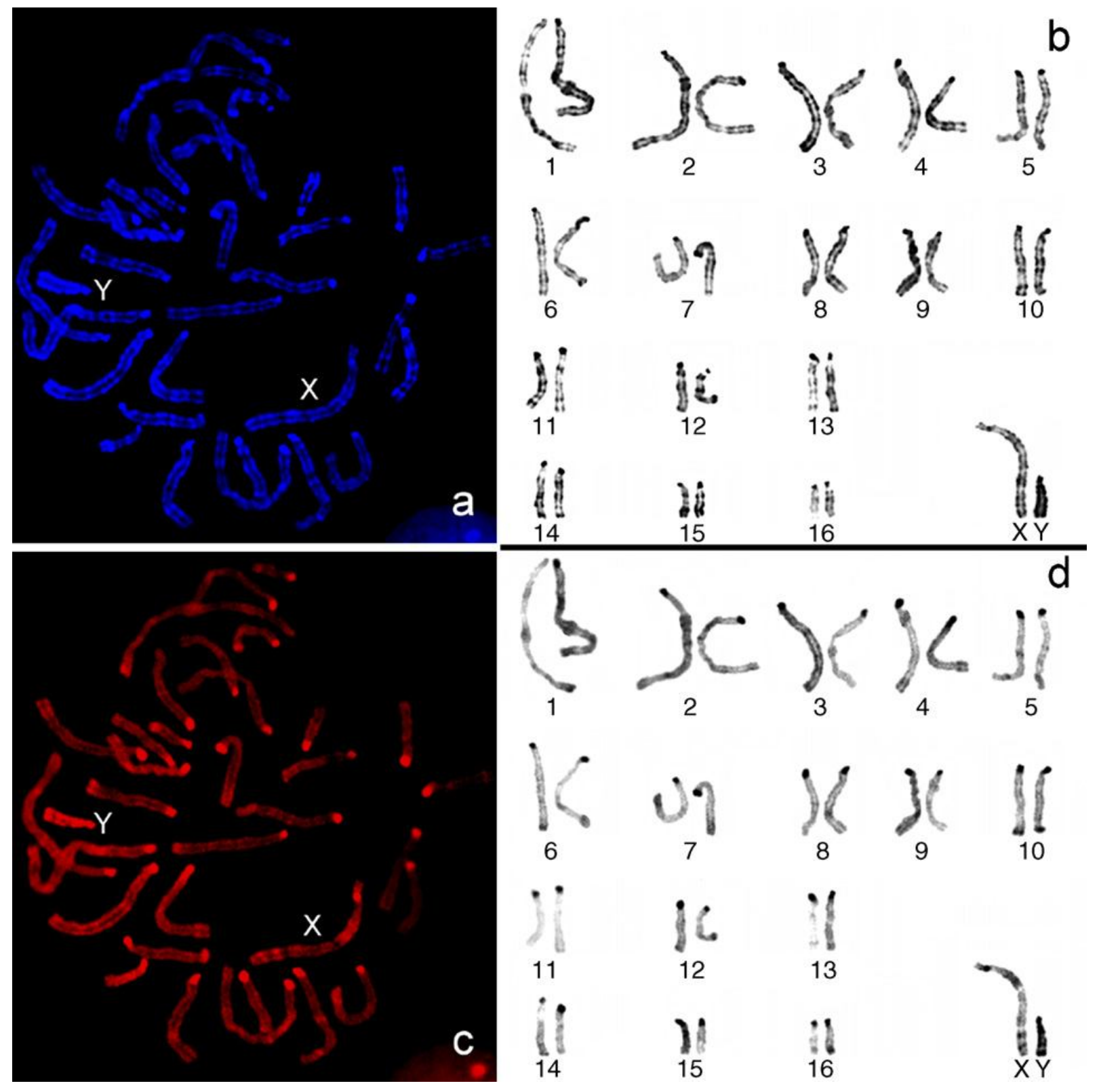

Fig. 1. This figure presents a demonstrative metaphase (a and c) and respective karyotypes (b and d) from Praomys tullbergi (male, $2 \mathrm{n}=34$ ) of the sequential procedures used in this work for the identification of chromosomes: GTD-banding (a and b) and CBPbanding treatment (c and d). The sex chromosomes are indicated in the images (a and c). 


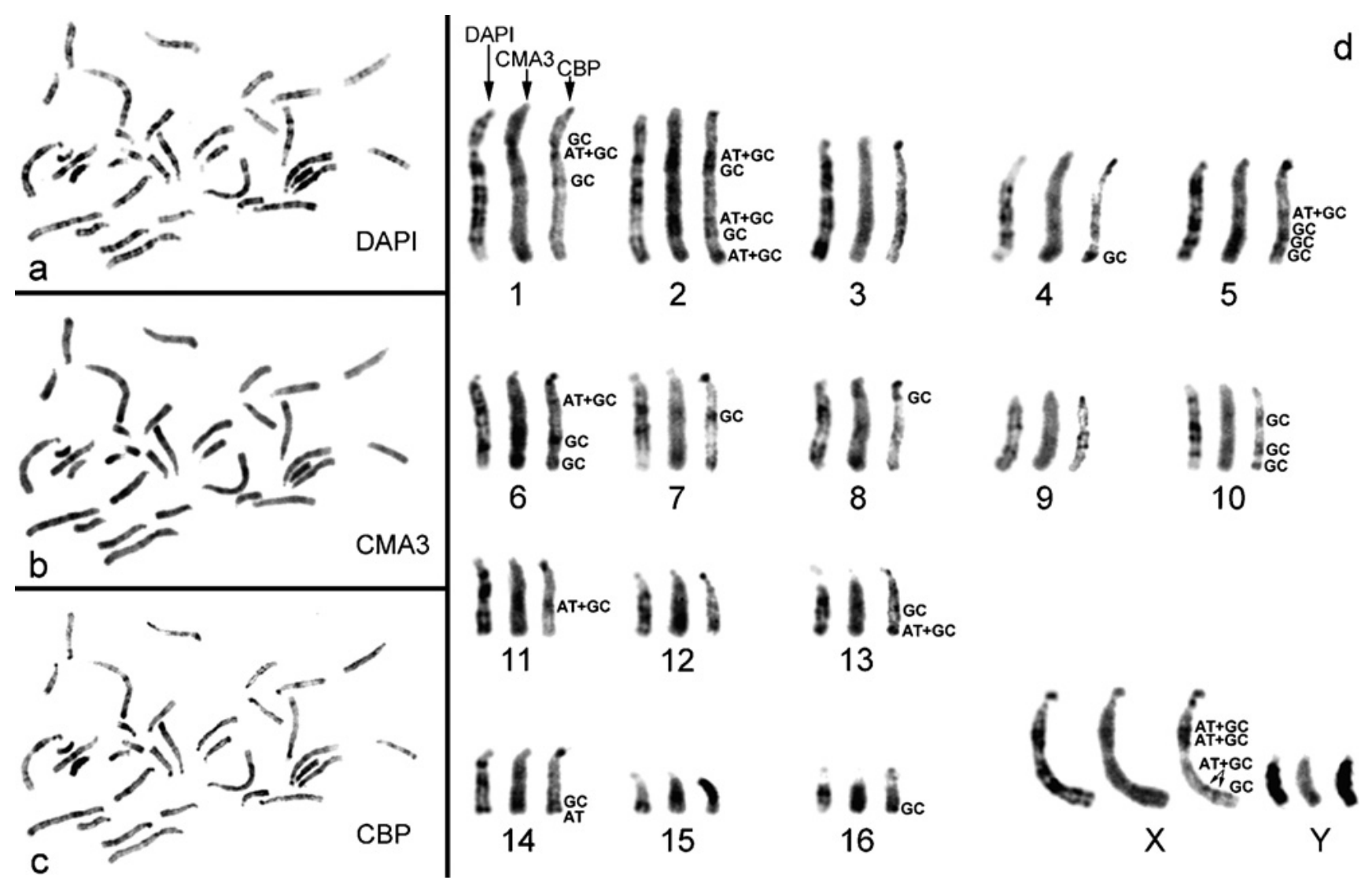

Fig. 2. The application of DNA-specific fluorescent dyes was performed in order to ascertain the affinity to AT or GC-rich regions in P.tullbergi karyotype. A metaphase from P.tullbergiwith the sequential treatments (DAPI + CMA3 + CBP) is showed (a, b and c, respectively). In d it is presented the haploid karyotype of the three treatments for an improved comparative analysis. Examples of the different constitutive heterochromatin regions molecular composition are indicated in the image (AT, GC, AT + GC). 


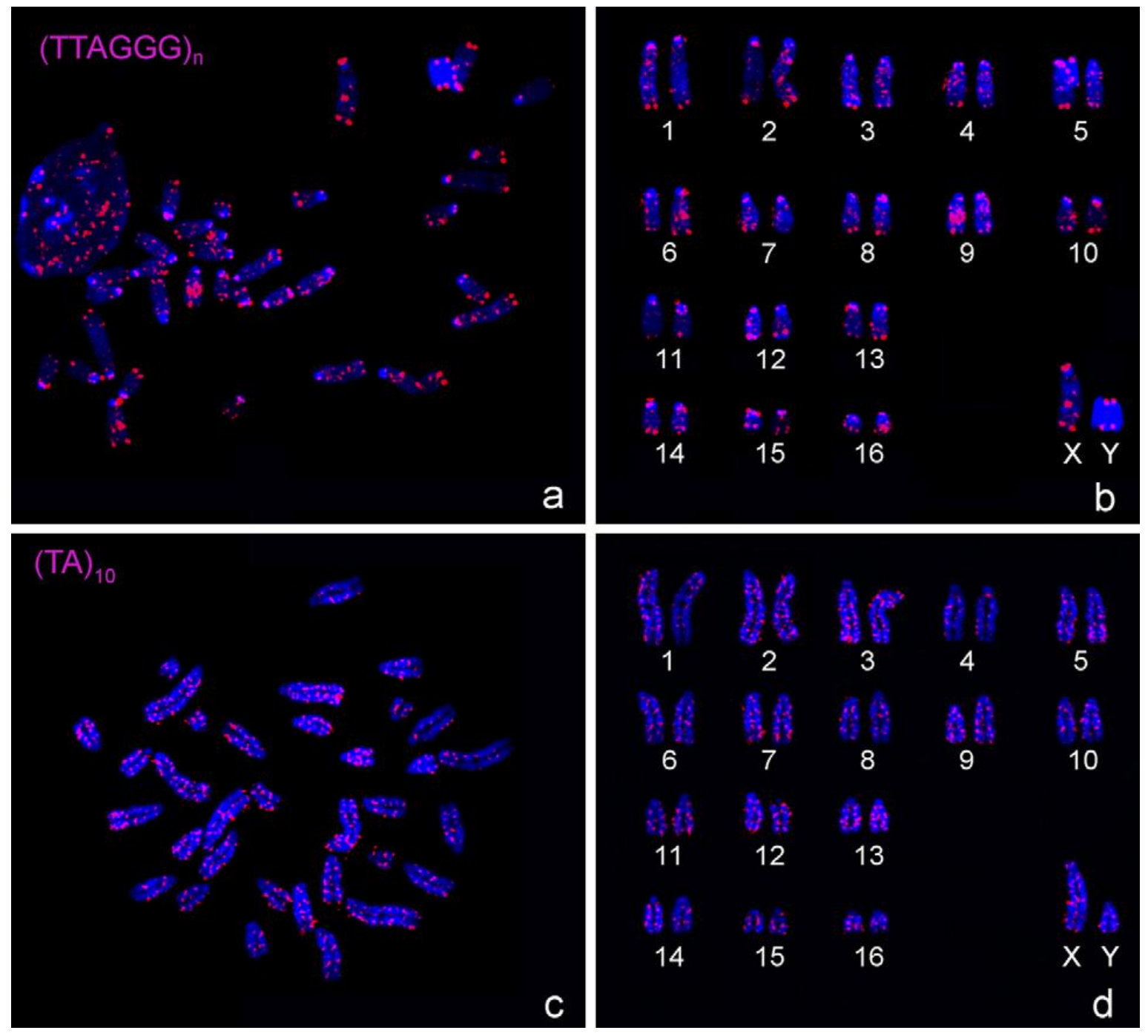

Fig. 3. In situ hybridization of (a) telomeric DNA sequences (TTAGGG) $)_{n}$ and (c) oligonucleotide (TA) $)_{n}$ probes (both probes were detected with anti-digoxigenin- rhodamine, presented in red colour) to metaphase chromosomes of $P$. tullbergi (chromosomal DNA was stained with DAPI, presented in blue colour). In b and $\mathrm{d}$ are shown the karyotypes of the metaphases a and c, respectively. 

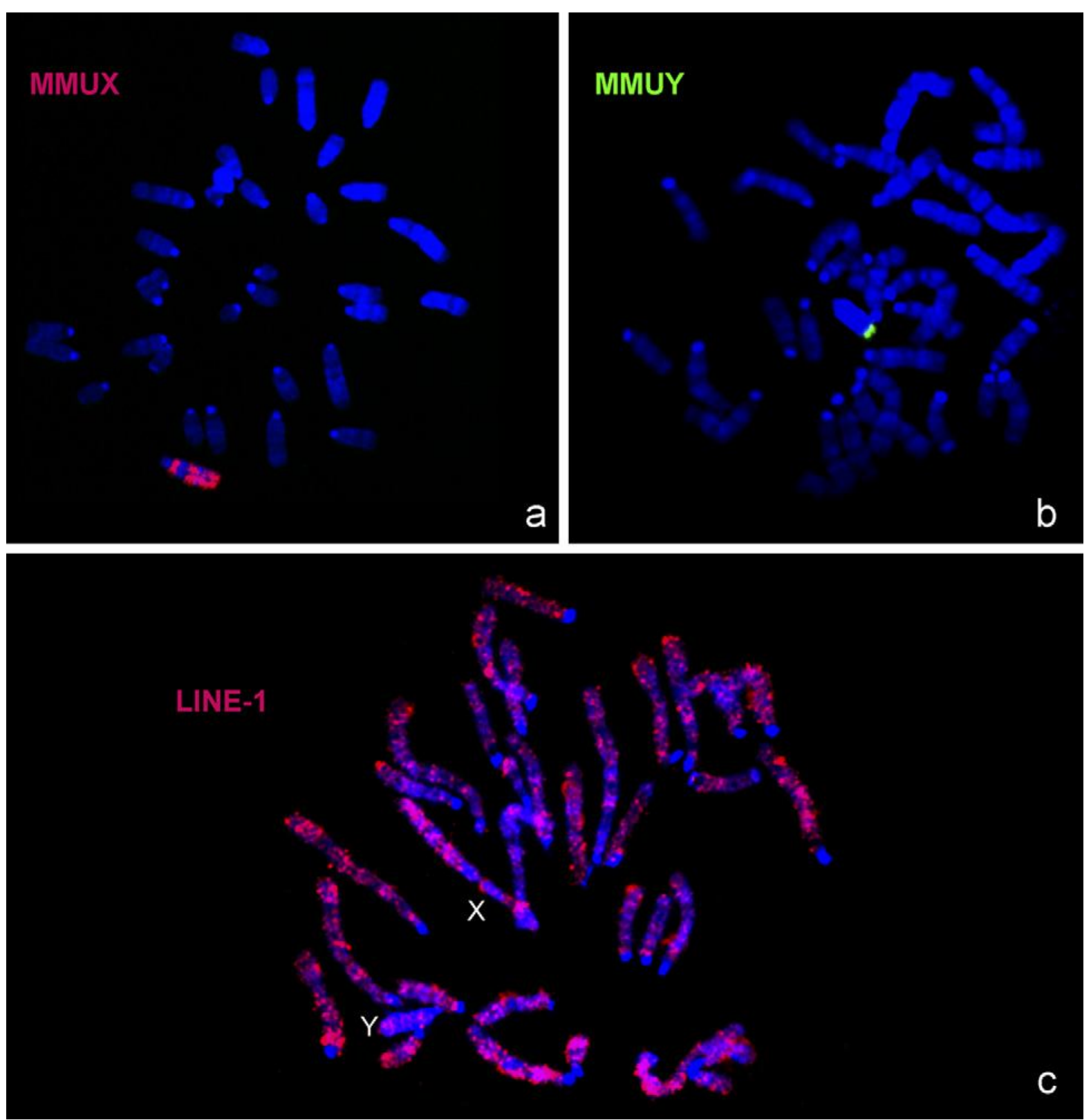

Fig. 4. In situ hybridization of (a) MMUX (detected with anti-digoxigenin-rhodamine, presented in red colour) and (b) MMUY (detected with FITC conjugated with avidin, presented in green colour) paint probes to metaphase chromosomes of $P$. tullbergi (chromosomal DNA is stained with DAPI, presented in blue colour). Representative in situ hybridization of LINE-1 probe in $P$. tullbergi chromosomes is showed in c. This probe was detected with anti-digoxigenin-rhodamine (red colour) and the $P$. tullbergi chromosomes were stained as described above. The sex chromosomes are indicated. 

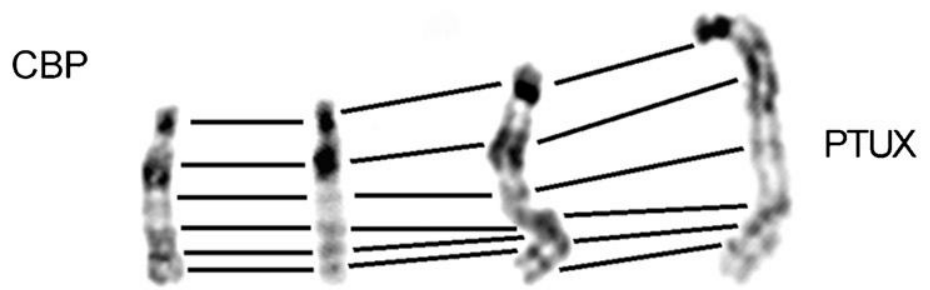

a
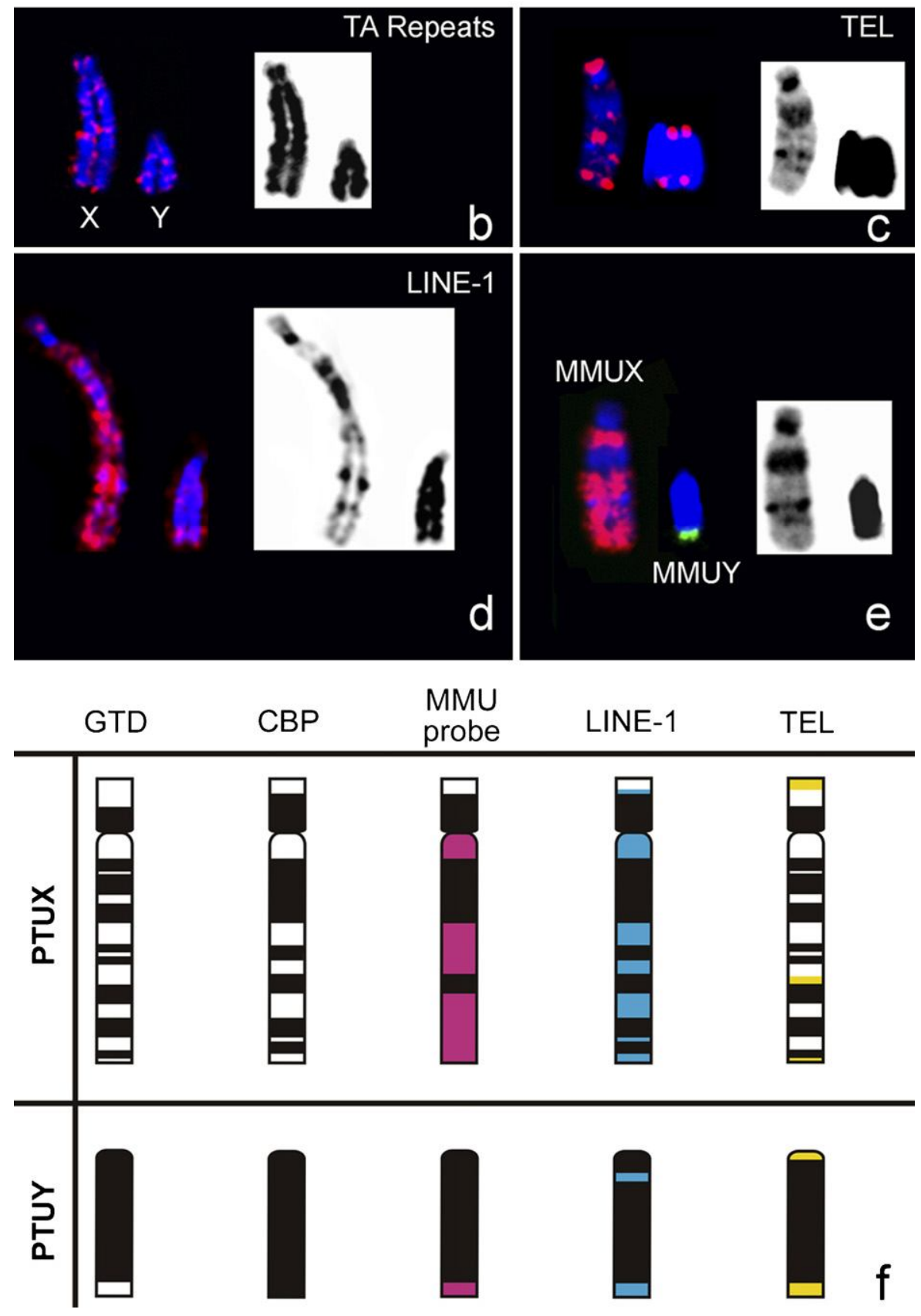

Fig. 5. In this figure is presented a compilation of all the data obtained for the P.tullbergisex chromosomes (a-e) and the respective ideograms (f). In a, the CBP- banding on the $\mathrm{X}$ chromosome is presented for different chromosome condensation levels; is important to note that the C-bands detected are similar. From b to e the sex chromosomes are presented but only indicated in image $\mathrm{b}$. Finally, in $\mathrm{f}$ the physical localization of the different probes to the sex chromosomes are presented in different colours: red (MMU probes), blue (LINE-1) and yellow (telomeric DNA sequences-TEL). 